\title{
Using direct and indirect measures to study perception without awareness
}

\author{
EYAL M. REINGOLD and PHILIP M. MERIKLE \\ University of Waterloo, Waterloo, Ontario, Canada
}

\begin{abstract}
Many studies directed at demonstrating perception without awareness have relied on the dissociation paradigm. Although the logic underlying this paradigm is relatively straightforward, definitive results have been elusive in the absence of any general consensus as to what constitutes an adequate measure of awareness. We propose an alternative approach that involves comparisons of the relative sensitivity of comparable direct and indirect indexes of perception. The only assumption required by the proposed approach is that the sensitivity of direct discriminations to relevant conscious information is greater than or equal to the sensitivity of comparable indirect discriminations. The proposed approach is illustrated through an evaluation of Avant and Thieman's (1985) recent claim that an indirect measure of perception based on judgments of apparent visual duration provides a more sensitive indicator of perception than does a direct measure based on forced-choice recognition. Contrary to this claim, when direct and indirect indexes are measured under comparable conditions, an indirect measure based on judgments of perceived duration provides a less sensitive index of perceptual processing than do comparable direct measures. The proposed approach provides a general conceptual/methodological framework for using the dissociation paradigm in studies directed at establishing unconscious processes.
\end{abstract}

Many studies directed at demonstrating perception without awareness have relied on the dissociation paradigm (see Erdelyi, 1985, 1986). The basic logic underlying this paradigm is that perception without awareness can be demonstrated via a dissociation between two indexes of perceptual processing. One index is assumed to indicate the availability of stimulus information to awareness or consciousness, and the second is assumed to indicate the availability of stimulus information, independent of whether or not this information is available to consciousness. In the frequently employed version of the dissociation paradigm, such as the one advocated by Holender (1986), a demonstration of perception without awareness requires unequivocal evidence that stimulus information that is completely unavailable to awareness, as indicated by a direct measure of perception, is nevertheless perceived and capable of influencing higher-level decision processes, as indicated by a second, typically indirect, index of perceptual processing.

This version of the dissociation paradigm has three methodological requirements or criteria that must be satisfied before perception without awareness is demonstrated. First, an adequate direct measure of the perceptual infor-

This research was supported by Grant APA-231 from the Natural Sciences and Engineering Research Council of Canada to the second author. Jan Williams deserves thanks for developing the software and for keeping the hardware in working order, and Sherri Van Velzer and Elizabeth Moore deserve thanks for the cheerful manner in which they tested the subjects. We thank Elizabeth Bosman for her many helpful comments on earlier drafts of the paper. Correspondence concerning this article should be addressed to either Eyal M. Reingold or Philip M. Merikle, Department of Psychology, University of Waterloo, Waterloo, Ontario N2L 3G1, Canada. mation available to consciousness or awareness must be selected. Second, this measure of conscious perceptual experience must be shown to indicate null sensitivity. Finally, given that the measure of conscious awareness indicates null sensitivity, the second measure of perceptual processing must be shown to have greater than zero sensitivity.

Although the logic underlying this widely used version of the dissociation paradigm is relatively straightforward, extensive experimental work conducted within the framework of this paradigm has not provided any definitive answers as to the validity of perception without awareness. In fact, as indicated by Holender's (1986) recent review and associated peer commentaries, the controversy over perception without awareness is far from resolved. Much of this continuing controversy centers on two methodological requirements of the dissociation paradigm. First, there is no general consensus as to what constitutes an adequate direct measure of conscious perceptual awareness. Second, the dissociation paradigm, as it is typically used, requires any assumed measure of conscious awareness to indicate null sensitivity, or, in other words, null awareness. Given the extreme methodological difficulties in establishing null sensitivity for any measure of perception (e.g., Macmillan, 1986), it is perhaps not surprising that most studies can be criticized for failing to demonstrate null awareness convincingly (e.g., Holender, 1986; Merikle, 1982).

In the absence of any general agreement as to what constitutes an adequate measure of conscious awareness, the general strategy in studies of perception without awareness has been to select a measure of conscious perceptual processing that is "intuitively" reasonable. Thus, mea- 
sures as diverse as forced-choice presence-absence decisions (e.g., Balota, 1983; Fowler, Wolford, Slade, \& Tassinary, 1981; Marcel, 1983), forced-choice discriminations among a small number of stimulus alternatives (e.g., Cheesman \& Merikle, 1984, 1985, 1986; Purcell, Stewart, \& Stanovich, 1983), stimulus identification (e.g., McCauley, Parmelee, Sperber, \& Carr, 1980), and even left-right discriminations (Greenwald \& Liu, 1985) have been used to index conscious perceptual processing. The only common characteristic shared by these various tasks is that they provide direct measures of perceptual processing; that is to say, subjects are explicitly instructed to perform the discriminations of interest. However, without a general theoretical framework and sufficient empirical data to guide the selection of measures, it is impossible to know if these different measures of conscious awareness are either adequate or comparable (see Duncan, 1985; Erdelyi, 1986).

Any application of the dissociation paradigm to the study of perception without awareness is problematic unless these issues concerning what constitutes an adequate measure of conscious perceptual experience are resolved. In the present paper, we propose an alternative approach to the study of perception without awareness that we believe overcomes many of the difficulties inherent in the dissociation paradigm as it is commonly used. In particular, we propose that comparisons between the relative sensitivity of direct and indirect measures have the potential to provide considerable information relevant to questions concerning the relation between consciousness and perception, if these comparisons are made under appropriate conditions. Accordingly, we first discuss the empirical distinction between direct and indirect measures, and then discuss assumptions concerning how direct and indirect measures may be related to conscious and unconscious perceptual processes. In addition, we outline an ideal set of methodological criteria that should be satisfied when comparisons between direct and indirect measures are made. Finally, we illustrate the proposed approach by evaluating a recent claim by Avant and Thieman (1985) that judgments of apparent visual duration provide a sensitive indirect measure of unconscious perception.

\section{Defining Direct and Indirect Measures}

As a first step in comparing the relative sensitivity of direct and indirect measures, we propose that the terms direct and indirect should refer only to characteristics of experimental tasks. More formally, discriminations among a set of alternative stimulus states (e.g., S1, S2, ... Sn) should be considered a direct measure of perception if the discriminative response is part of the task definition, as expressed in the instructions given to the subjects. Conversely, if the discriminative response is not part of the task definition, it should be considered an indirect measure of perception. For example, consider two possible tasks that measure whether or not the words in compound color-word stimuli (Stroop,1935) are perceived. If subjects are instructed to identify which word from a known set of the words was presented, then performance on this task would constitute a direct measure of word perception, as the instructions explicitly require word identification. However, if subjects are instructed to identify the colors, then any effect that the words may have on color-naming performance would constitute an indirect measure of word perception, since the subjects are not instructed to respond to the identity of the words.

The proposed distinction between direct and indirect measures is entirely consistent with the manner in which these terms have been used previously (e.g., Fowler et al., 1981; Humphreys, 1981; Marcel, 1983). However, what characterizes the proposed distinction is the explicit limitation of the terms direct and indirect to descriptions of experimental tasks. Thus, the proposed distinction is based solely on empirical considerations; consequently, the direct/indirect distinction, by itself, has no implications as to the underlying processes that may influence performance on each type of task.

\section{A Priori Assumptions}

In the context of the dissociation paradigm, any comparisons between direct and indirect indexes of perception necessarily involve a priori assumptions concerning how direct and indirect indexes may be related to conscious and unconscious processes (see Erdelyi, 1985, 1986). Unfortunately, these assumptions are rarely made explicit. Indeed, much of the long-standing controversy over perception without awareness revolves around differences in the implicit assumptions adopted by different investigators (see Bowers, 1984, for a related discussion). In this section, we initially review several approaches which imply that conscious perception can be operationally defined solely in terms of the sensitivity of direct measures of perception. We then propose an alternative approach based on an assumption concerning the relative sensitivity of comparable direct and indirect indexes to conscious perceptual information.

Sensitivity of direct measures: The exclusiveness assumption. Holender's (1986) position requires the most stringent set of assumptions concerning how direct measures of perception are related to conscious processes. Rather than distinguishing direct and indirect indexes of perception on the basis of task characteristics, he suggested that direct and indirect indexes should be distinguished on the basis of an observer's intentions. After considering a number of direct measures used to indicate awareness-for example, forced-choice detection and forced-choice discrimination-Holender stressed that "one property common to all these indicators of awareness is that subjects make their responses intentionally. It is fundamental that an indicator of awareness must be intentional"' (p. 51). Consequently, "any voluntary discriminative response that can be elicited on the basis of the meaning of a stimulus will be considered direct evidence for semantic activation" (p. 1), and "direct evidence of semantic activation is in itself taken as evidence for conscious identification"' (p. 51). Thus, according to 
Holender, all direct measures of perception are by definition indexes of intentional, and therefore conscious, discriminations.

Although never clearly stated by Holender, it logically follows from his position that direct measures of perception exclusively index conscious perceptual processes or, conversely, that direct measures of perception are never influenced by unconscious perceptual processes. This exclusiveness assumption implies that a sufficient condition for demonstrating perception with awareness is any evidence indicating that a direct measure shows greater than zero sensitivity.

The exclusiveness assumption is questionable, and an equally plausible assumption has been suggested by Marcel (1983). According to Marcel, it is entirely possible that

In attempting to make deliberate judgements based on information of whose external source one is unaware, it would seem that one makes use of the relevant nonconscious information, if it is available, by relying passively on its effects (e.g. upon attention) rather than being able selectively to retrieve it or be sensitive to it such that it can be the basis of an intentional choice. (p. 211)

Thus, for Marcel, intentional responding is based on subjective phenomenal awareness; however, neither subjective phenomenal awareness nor intentional responding are necessarily indexed by a direct measure of perception.

Even though Marcel (1983) and Holender (1986) agree that intentionality is a critical characteristic of consciousness or awareness, they disagree completely as to the relation between intentionality and direct discriminative responses. Marcel's position is consistent with an increasing number of proposals suggesting that any definition of perceptual awareness must be based on a consideration of an observer's subjective phenomenal experience when performing a task, and not on the characteristics of the task per se (e.g., Cheesman \& Merikle, 1985; Dixon, 1971, 1981; Fowler, 1986; Henley, 1984; Merikle \& Cheesman, 1986; Navon, 1986; Paap, 1986; Wolford, 1986). Holender's position, on the other hand, implies that all direct discriminative responses necessarily reflect intentional responding. The problem with Holender's position is that the intentions of an observer cannot be directly observed or measured. Therefore, any inference concerning an observer's intentions when performing tasks that require direct discriminations, especially tasks requiring forced-choice discriminations, is problematic. If an observer's intentions cannot be measured unambiguously, then equating the distinction between direct and indirect indexes of perception with a distinction between voluntary and involuntary actions cannot be justified.

Sensitivity of direct measures: The exhaustiveness assumption. Although many researchers may agree that the exclusiveness assumption is unwarranted, it is important to consider another implicit assumption underlying many recent applications of the dissociation paradigm. Investigators applying the dissociation paradigm often as- sume that null awareness is demonstrated when the direct measure used in their study indicates null perceptual sensitivity. This assumption implies that greater than zero sensitivity on a direct measure is a necessary condition for demonstrating conscious perception. In other words, the approach adopted in many studies implies that whenever conscious perceptual processes occur, direct discriminations will show greater than zero perceptual sensitivity. This implied consequence of conscious perception can be true only if it is also assumed that direct measures provide an exhaustive measure of conscious perceptual experience. If this additional exhaustiveness assumption is not made, then there is no reason to expect that a direct measure of perception will always indicate greater than zero perceptual sensitivity, given conscious perceptual experience.

Serious objections can be raised regarding the validity of this implied exhaustiveness assumption. First and foremost is the problem in establishing which direct measures of perception qualify as exhaustive measures of consciousness. Different direct measures have been used in different studies, and certain direct measures may be more sensitive than other direct measures to conscious perceptual information. Furthermore, since any direct measure requires an observer to respond on the basis of a particular stimulus dimension, the different direct measures that have been used to index conscious perception require different discriminations based on different types of taskrelevant information (Duncan, 1985; Navon, 1986). In light of these concerns, one might be tempted to select the direct index with the lowest "absolute threshold" as an exhaustive measure of conscious perceptual experience. However, if one rejects the exclusiveness assumption, and therefore accepts the possibility that a direct measure is potentially sensitive to unconscious perceptual information, such an extremely conservative procedure might preclude perception without awareness from ever being demonstrated (see Bowers, 1984).

Given these unresolved issues concerning how direct indexes of perception may be related to conscious and unconscious processes, it is difficult to justify either the exclusiveness assumption or the exhaustiveness assumption on an a priori basis. More generally, as long as these issues remain unresolved, it may be difficult to ever justify any a priori assumptions concerning the relation between conscious perception and the absolute sensitivity of direct indexes. For this reason, we propose an alternative approach to the study of unconscious perception based on the relative sensitivity of direct and indirect measures to conscious perceptual information.

Relative sensitivity of direct and indirect measures to conscious information. By considering the relative sensitivity of direct and indirect indexes to conscious perceptual information, it is possible to use the dissociation paradigm to study unconscious perception and to make only one a priori assumption. Obviously, it is desirable to minimize a priori assumptions, and we propose that 
only the following reasonable, but minimal, working assumption is required by the logic of the dissociation paradigm:

The sensitivity of a direct discrimination is assumed to be greater than or equal to the sensitivity of a comparable indirect discrimination to conscious, taskrelevant information.

This assumption is based on the reasonable supposition that instructions directing an observer to respond on the basis of a particular stimulus dimension should optimize the observer's use of conscious information that may be available concerning this dimension. At the very most, indirect and direct measures may be equally sensitive to relevant conscious perceptual information; however, an indirect measure should never provide a better index of conscious perceptual experience than is provided by a comparable direct measure.

It is important to emphasize what is not assumed or implied by the proposed approach. Most critically, when a direct measure of perception indicates greater than zero sensitivity, this finding by itself is not assumed to be either a sufficient condition (i.e., the exclusiveness assumption) or a necessary condition (i.e., the exhaustiveness assumption) for demonstrating conscious perception. Such conclusions do not fallow from the proposed assumption, since the assumption does not impose any constraints on the absolute sensitivity of direct and indirect indexes. Rather, the proposed assumption only constrains the relative sensitivity of direct and indirect measures to conscious, task-relevant information. Thus, given this minimal constraint, the sensitivity of direct and indirect indexes can potentially reflect the availability of conscious, unconscious, or both conscious and unconscious perceptual information.

\section{Interpretation of Possible Patterns of Results}

The important implication of the above assumption is that unconscious perception is demonstrated whenever an indirect measure shows greater absolute sensitivity than does a comparable direct measure to a particular stimulus dimension. Since this conclusion might not be immediately apparent, it is deduced step by step in the appendix. Other patterns of results are certainly possible. However, given the logic behind comparing the relative sensitivity of direct and indirect measures, these other possible patterns of results would not provide any definitive evidence. For example, if an indirect index is less sensitive to a particular stimulus dimension than is a comparable direct index, this pattern of results would provide no evidence either for or against unconscious perception. This is the case, since it cannot be deduced whether the sensitivity of either the direct or the indirect indexes reflects conscious, unconscious, or both conscious and unconscious perceptual processes. Thus, the proposed approach provides a method for demonstrating perception without awareness, but it is ineffective as a method for unequivocally demonstrating perception with awareness. This is a direct consequence of the absence of a valid measure of conscious perceptual experience.

\section{Methodological Criteria}

A critical aspect of the proposed assumption concerning the relative sensitivity of direct and indirect measures to conscious perceptual experience is the assertion that the sensitivity of both indexes should be measured under comparable experimental conditions. Otherwise, any observed dissociations between indexes may reflect only methodological differences and not a true difference in the relative sensitivity of these measures to conscious and unconscious perceptual processes. In this section, we review four methodological concerns that are relevant whenever comparisons are made between the relative sensitivity of direct and indirect measures.

1. Perceptual sensitivity versus response bias. Both direct and indirect indexes should be based on measures of discriminative responding that allow perceptual sensitivity to be assessed independent of any possible influences contributed by observer biases. Merikle (1982) discussed the interpretive problems that can arise when measures do not permit a clear distinction between sensitivity and bias. Although tasks based on forced-choice, discriminative responses are ideal for satisfying this criterion, other tasks, based on reaction time or physiological measures (e.g., the galvanic skin response [GSR]), could be used under appropriate conditions (see Dulany \& Eriksen, 1959).

2. Stimulus states. Direct and indirect indexes should measure discriminative responses among the same set of alternative stimulus states. Thus, a direct measure of discrimination among the stimulus states $S 1, S 2, \ldots, S n$ should always be compared with an indirect measure of the same discrimination. As noted by Duncan (1985), this condition has not been met in a number of recent studies (e.g., Balota, 1983; Fowler et al., 1981; Marcel, 1983). In these studies, the direct measure of perceptual discrimination was based on presence/absence decisions (i.e., S1, $S 2, \ldots$, or $\mathbf{S} n$ versus a null stimulus state), whereas the indirect measure, priming, was based on discriminations among the stimulus alternatives (i.e., S1 vs. S2 ... vs. $\mathrm{S} n$ ). If direct and indirect indexes do not measure completely equivalent discriminations under comparable conditions, then any dissociation between these indexes may simply reflect differences in either (1) the availability of the relevant stimulus information or (2) the difficulty of the required discrimination.

3. Task context. Both direct and indirect measures should index stimulus discriminations under display conditions that are comparable except for the direct/ indirect instruction. If display conditions are not comparable across measures, then any observed differences in sensitivity between measures may simply reflect a difference in either the encoding or the retrieval environment. For example, Purcell et al. (1983) showed that differential dark adaptation caused by slightly different display conditions can account for McCauley et al.'s (1980) findings 
indicating that an indirect priming measure is a more sensitive indicator of perception than is a direct identification measure. Likewise, Bernstein, Vyas, Bissonnette, and Barclay (1987) showed that the sensitivity of a direct measure of stimulus discrimination can depend critically on both instructional bias and the context provided by the presentation of a second, related stimulus. These considerations indicate that every effort should be made to ensure that direct and indirect indexes are measured under completely comparable display conditions.

4. Response metric. Direct and indirect indexes should be based on the same response metric. For example, one of Eriksen's (1956, 1960) classic criticisms of the subception effect reported by Lazarus and McCleary (1951) is that the GSR and the perceptual identification measures were not based on the same metric. According to Eriksen, Lazarus and McCleary's finding that the GSR exceeds baseline even on those trials in which a critical stimulus is not correctly identified may very well be an artifact of the discrete measurement of perceptual identification versus the continuous measurement of the GSR. Eriksen's criticism is also applicable to recent priming studies in which the continuous reaction time measure was compared with a discrete verbal response measure. Thus, if direct and indirect indexes are not based on the same metric, any observed dissociation between these measures may simply reflect an artifact that arises when two different measurement scales are compared.

Although an ideal experiment comparing the relative sensitivity of direct and indirect measures is one in which both indexes are measured under conditions that are equivalent in terms of the above methodological criteria, in practice, a series of converging experiments may be required. In any case, the above criteria provide critical guidelines for the evaluation and interpretation of any demonstrated dissociations between direct and indirect measures of perception.

\section{Advantages of the Proposed Approach}

Relative to previous approaches, the proposed approach to the study of perception without awareness has two major advantages.

Conceptual advantage. As discussed above, since inferences concerning unconscious processes are based on a consideration of the relative sensitivity of direct and indirect measures, rather than on a consideration of the absolute sensitivity of direct measures, there is a significant reduction in a priori assumptions. As a consequence, the proposed approach bypasses the controversy over the measurement of awareness and, at the same time, provides a method for studying perception without awareness.

Methodological advantage. To demonstrate unconscious perception, it is sufficient to establish that the sensitivity of the indirect measure is greater than the sensitivity of a comparable direct measure, even if the sensitivity of the direct measure is greater than zero. Thus, by following the proposed approach, the establishment of null awareness is no longer a prerequisite for demonstrating perception without awareness.

\section{Illustration: Dulany and Eriksen (1959)}

One study that illustrates how the proposed methodological criteria can be successfully applied was reported by Dulany and Eriksen (1959). These investigators compared the relative sensitivity of two measures of perceptual processing: a direct verbal discriminative response and an indirect discriminative response based on the GSR. The task for the observers was to indicate verbally whether a test light, which could vary in brightness, appeared during the first or second of two successive 3-sec intervals. In addition, since a reliable GSR to a standard light stimulus was established prior to the discrimination task, a GSR "choice" was recorded on each trial by noting the test interval in which the larger GSR occurred. These procedures satisfy the four proposed methodological criteria for comparing direct and indirect indexes: both indexes (a) measured discriminative responses between the same alternative stimulus states (Criterion 2) under identical display conditions (Criterion 3), and (b) were based on twoalternative forced-choice tasks that allowed perceptual sensitivity to be measured in terms of a common metric (Criterion 4) independent of possible observer biases (Criterion 1).

When Dulany and Eriksen (1959) compared the relative sensitivity of the direct and indirect measures, they found that the direct verbal discriminative response was considerably more sensitive for most observers than the indirect GSR measure. According to the logic of the dissociation paradigm, these results provide no evidence to indicate that GSR discriminations are mediated by unconscious perceptual information that is inaccessible to awareness. On the other hand, it is also important to emphasize that the logic of the proposed approach does not lead to the conclusion that the greater sensitivity of the direct measure necessarily indicates that brightness discriminations were mediated by conscious perceptual processes. As previously noted, direct and indirect measures might index conscious, unconscious, or both conscious and unconscious perceptual processes. Thus, whenever a direct measure is found to be more sensitive than an indirect measure, the logic of the proposed approach does not lead to any conclusive answers concerning the possible influence of unconscious perceptual processes.

\section{Application: Judgment of Apparent Visual Duration as an Indirect Measure}

Given the proposed methodological criteria, there are certain limitations as to the direct and indirect measures that can be used successfully to investigate the relation between consciousness and perception. However, a recent series of experiments reported by Avant and Thieman (1985) suggests a possible approach. These investigators claimed to have demonstrated that judgments of apparent visual duration provide an indirect measure of 
perceptual processing that is more sensitive to the lexical status of a visual stimulus than are direct discriminative responses. This claim is based on results indicating that judgments of perceived visual duration are influenced by the familiarity of a stimulus (i.e., word vs. nonword) even though direct discriminative responses among the stimuli approximate a chance level of accuracy. If Avant and Thieman's claim is correct, then judgment of apparent visual duration would provide a useful indirect measure to study the relation between consciousness and perception within the conditions specified by the proposed approach.

Unfortunately, Avant and Thieman's (1985) results cannot be considered definitive, because temporal duration judgments and direct discriminative responses were measured under considerably different conditions. Observers judged temporal duration by indicating which of two successively presented displays appeared to last longer. On the other hand, direct discriminative responses were based on a forced-choice recognition task in which a stimulus was presented, and observers were then shown two alternative displays and required to choose the display that had just been presented. These procedures do not satisfy either Criterion 2 or Criterion 3, because the direct and indirect indexes measured discriminative responding between different stimulus states under different display conditions. Thus, the observed differences between the two indexes may simply reflect differences in the difficulty of the required discrimination. Therefore, Avant and Thieman's results cannot be considered definitive.

The major purpose of the present study was to compare, under conditions that satisfy the four proposed methodological criteria, temporal duration judgments with direct measures of perceptual processing based on present/absent and word/nonword discriminations. Since temporal duration judgments do not require any discrimination regarding stimulus content, the time judgment task has the potential to provide an excellent indirect measure of perceptual processing when it is contrasted to direct discriminative judgments measured under precisely the same conditions. Accordingly, Experiments 1 and 2 were designed to replicate well-established findings indicating that filled intervals are perceived as longer than empty intervals of the same duration (e.g., Allan, 1979; Craig, 1973; Goldfarb \& Goldstone, 1963) and that perceived duration is influenced by stimulus familiarity (e.g., Avant \& Lyman, 1975; Avant, Lyman, \& Antes, 1975; Avant \& Thieman, 1985; Devane, 1974; Warm, Greenberg, \& Dube, 1964; Warm \& McCray, 1969; Witherspoon \& Allan, 1985). In Experiments 3 and 4, the sensitivity of temporal duration judgments, as an indirect measure of perceptual processing, was compared with the sensitivity of direct word/nonword and presence/absence discriminations.

\section{EXPERIMENT 1}

The major purpose of this experiment was to replicate, with our methodology, two findings demonstrating that nontemporal factors influence the perceived duration of equivalent temporal intervals. One phenomenon, commonly referred to as the filled-duration illusion, is that filled intervals are judged as longer than empty intervals of the same temporal duration (e.g., Allan, 1979; Craig, 1973; Goldfarb \& Goldstone, 1963). A second finding is that judgments of temporal duration are influenced by stimulus familiarity (e.g., Avant \& Lyman, 1975; Avant et al., 1975; Devane, 1974; Warm et al., 1964; Warm \& McCray, 1969; Witherspoon \& Allan, 1985). Both findings demonstrate the influence of nontemporal factors on duration judgments and suggest that judgments of temporal duration have the potential to provide a sensitive indirect measure of perception.

Although the filled-duration illusion is well established, the expected direction of any effect of stimulus familiarity upon perceived duration is problematic. Avant and his colleagues (e.g., Avant \& Lyman, 1975; Avant et al., 1975) reported that intervals in which words are presented are judged to be shorter than intervals in which nonwords are presented. In contrast, other investigators (e.g., Devane, 1974; Warm \& McCray, 1969; Witherspoon \& Allan, 1985) found that familiar words are perceived as longer in duration than unfamiliar words. Thus, depending upon the particular study that is considered, evidence can be found indicating either a positive or a negative relation between stimulus familiarity and perceived duration.

In general, studies finding positive correlations can be distinguished from studies finding negative correlations in terms of both the judgment task and the definition of stimulus familiarity. More specifically, Avant and his colleagues (Avant \& Lyman, 1975; Avant et al., 1975) found a negative correlation between stimulus familiarity and perceived duration when stimulus familiarity was defined in terms of a word/nonword distinction and perceived duration was measured by a comparative judgment task. In contrast, studies in which the familiarity of English words was varied on the basis of either word frequency (e.g., Devane, 1974; Warm \& McCray, 1969) or stimulus repetition (Witherspoon \& Allan, 1985) indicate that perceived duration and stimulus familiarity are positively correlated. In addition, studies demonstrating positive correlations typically involve absolute or categorical judgments rather than comparative judgments. Given these methodological differences, it has been suggested that either the operational definition of familiarity (Witherspoon \& Allan, 1985 ) or the type of judgment task (Thomas \& Weaver, 1975) may account for the conflicting patterns of results concerning the relation between perceived duration and stimulus familiarity.

In the present study, stimulus familiarity was based on a word/nonword distinction, and a comparative judgment task was used to measure perceived duration. These aspects of the method are similar to the methodology used by Avant and his colleagues. Therefore, if either the definition of familiarity or the type of judgment task are critical in determining the direction of the relation between stimulus familiarity and perceived duration, then these 
variables should be negatively correlated under the present experimental conditions.

\section{Method}

Subjects. Twenty undergraduate students at the University of Waterloo participated in the experiment. All subjects had normal or corrected-to-normal vision, and each subject was paid $\$ 10$ following completion of the experimental session.

Apparatus. All stimulus materials were displayed on an Electrohome color monitor that was interfaced to an Apple II + microcomputer via an Electrohome Supercolor board. The monitor was viewed through a hood that physically divided the screen into separate left-eye and right-eye fields, and each field was viewed through a rotating prism to aid fusion of the fields. The viewing distance was $65 \mathrm{~cm}$, and the luminance of each field measured $32 \mathrm{~cd} / \mathrm{m}^{2}$ when the light beige background color (Color No. 91) was displayed.

Three touch-sensitive plates were used by the subjects to initiate trial sequences and to indicate their decision following each trial. These plates were located on a table directly in front of the subjects. The plates were positioned in a manner that allowed each subject's hands to rest comfortably on the table. A plate on the left was used to initiate trials, and two plates on the right were used to indicate the decision following each presentation sequence.

Materials and Design. A pool of words containing 324 highfrequency nouns was compiled from the Kucera and Francis (1967) norms. Equal numbers of four-, five-, and six-letter words were selected, and the minimum word frequency was 57 occurrences/million. This pool of words provided the stimulus materials for the experimental trials administered to each subject.

Each of 432 experimental trials consisted of two different stimulus displays presented in succession. On 216 of these trials, one stimulus was a randomly selected word, and the other stimulus was a nonword variant of the same word. All nonwords were created by randomly rearranging the letters in words. On the remaining 216 trials, one stimulus was either a word or a nonword, and the second stimulus consisted of a blank field; word-blank and nonword-blank pairs each occurred on 108 trials.

The experimental trials were presented in six blocks of 72 trials. Within each trial block, word-nonword pairs were presented on 36 trials, and word-blank and nonword-blank pairs were each presented on 18 trials. In addition, in each trial block, the two possible presentation orders for each type of stimulus pair occurred equally often, and equal numbers of four-, five-, and six-letter stimuli were used with each stimulus pairing and presentation order combination. Within these constraints, the presentation order for the different conditions within each block of 72 trials was random.

All words and nonwords were presented binocularly in white (color no. 255) uppercase letters measuring $0.7 \mathrm{~cm}\left(0.6^{\circ}\right)$ horizontal $\times 1.1 \mathrm{~cm}\left(1.0^{\circ}\right)$ vertical. ${ }^{1}$ The length of these stimuli varied from $3.5 \mathrm{~cm}\left(3.1^{\circ}\right)$ for the four-letter words and nonwords to $5.3 \mathrm{~cm}$ $\left(4.7^{\circ}\right)$ for the six-letter words and nonwords. In addition, the masking stimulus, which was always presented binocularly immediately prior to the onset and immediately following the offset of each stimulus display, was a $9.0 \mathrm{~cm}\left(7.9^{\circ}\right) \times 1.5 \mathrm{~cm}\left(1.3^{\circ}\right)$ rectangular display consisting of randomly arranged letter pieces.

Procedure. Each subject was tested individually during a single session that lasted approximately $75 \mathrm{~min}$. Prior to the beginning of the experiment, ali subjects were instructed that they would see two "flashes" (i.e., stimulus displays) on each trial and that their task was simply to decide which "flash" was longer in duration. Absolutely no information was given to the subjects concerning the content of the stimulus displays.

Throughout the experiment, the masking stimulus was always present in the visual field except when the two stimulus displays were presented. Thus, the masking stimulus also served as the fixation stimulus, and the subjects were instructed to fixate this stimulus prior to initiating each trial. Once a trial was initiated, approximately 150 msec elapsed before the onset of the first stimulus display, and the interval between the offset of the first display and the onset of the second display was $1,000 \mathrm{msec}$. Each stimulus display was presented for $50 \mathrm{msec}$.

Following the offset of the second stimulus display, the subjects were required to decide which stimulus display was longer in duration. The subjects indicated their decision by pressing one of the two response plates located on the right side of the table. A minimum of 2,800 msec elapsed between the offset of the second stimulus display and the onset of a brief auditory signal indicating that the computer was ready to display the next trial sequence.

\section{Results and Discussion}

Mean proportions of "longer" responses for the wordblank and nonword-blank pairs are shown at the top of Table 1. As expected, filled intervals were judged as longer than blank intervals of the same stimulus duration for both word-blank pairs $[t(19)=12.0, p<.0001]$ and nonword-blank pairs $[t(19)=9.32, p<.0001]$. This finding represents a successful replication of the filledduration illusion. In addition, the type of filled interval (word vs. nonword) had only a marginal effect on judged duration $[t(19)=1.82, p<.10]$, with words being judged slightly more often than nonwords as longer than blank intervals.

The effect of stimulus familiarity (word vs. nonword) upon perceived duration is shown at the bottom of Table 1. The data for Experiment 1 are the mean proportions of "longer" responses for words and nonwords presented for $50 \mathrm{msec}$. As indicated in the table, a positive relation was found between stimulus familiarity and perceived duration. More specifically, words, in general, were judged as longer than nonwords $[t(19)=2.44$, $p<.05]$. Thus, the present results are entirely consistent with the results of many previous studies that demonstrated a positive relation between stimulus familiarity and perceived duration (e.g., Devane, 1974; Warm \& McCray, 1969; Witherspoon \& Allan, 1985).

On the other hand, the present results are completely inconsistent with the negative relation between stimulus familiarity and perceived duration reported by Avant and his colleagues (Avant \& Lyman, 1975; Avant et al., 1975; Avant \& Thieman, 1985). Given that we defined stimulus familiarity (word vs. nonword) in the same way that Avant and his colleagues did and used a similar comparative judgment task, neither factor can account for these

Table 1

Mean Proportions of "Longer" Responses in Experiments 1 and 2

\begin{tabular}{cccc}
\hline Experiment & Exposure Duration & \multicolumn{2}{c}{ Comparison } \\
\hline & & \multicolumn{2}{c}{ Word vs. Blank } \\
\cline { 3 - 4 } 1 & $50 \mathrm{msec}$ & .846 & .154 \\
1 & & Nonword vs. Blank \\
\cline { 3 - 4 } 2 & $50 \mathrm{msec}$ & .827 & .173 \\
1 & & \multicolumn{1}{c}{ Word vs. Nonword } \\
2 & $33 \mathrm{msec}$ & .503 & .497 \\
& $50 \mathrm{msec}$ & .541 & .459 \\
\hline
\end{tabular}


conflicting patterns of results. Thus, some other aspect of the methodology used by Avant and his colleagues must account for why they consistently find a negative relation between stimulus familiarity and perceived duration, while other investigators typically report a positive relation between these factors, similar to the one found in the present study.

\section{EXPERIMENT 2}

One difference between Experiment 1 and many of the studies reported by Avant and his colleagues is that we used a slightly longer exposure duration for the stimulus displays (i.e., $50 \mathrm{msec}$ instead of 30). Accordingly, Experiment 2 was designed to explore the influence of exposure duration on both the direction and the magnitude of the effect of stimulus familiarity upon perceived duration.

\section{Method}

Subjects. Twenty undergraduate students at the University of Waterloo participated in the experiment. All subjects had normal or corrected-to-normal vision, and each subject was paid $\$ 10$.

General. A total of 288 words was selected from the pool of words used in Experiment 1. The selected words had the higher word frequencies, so that the minimum word frequency for any word used in this experiment was 66 occurrences/million. In addition, equal numbers of four-, five-, and six-letter words were selected.

On all experimental trials, one stimulus display contained a randomly selected word and the other stimulus display contained a nonword variant of the same word. However, there were two types of experimental trials that differed in terms of the exposure duration used for the stimulus displays. On 144 trials, each stimulus display was presented for $33 \mathrm{msec}$, whereas on the remaining 144 trials, each display was presented for $67 \mathrm{msec}$.

The experimental trials were presented in 12 blocks of 24 trials. In each trial block, the displays were presented for $33 \mathrm{msec}$ on half the trials and 67 msec on the remaining trials. In addition, wordnonword and nonword-word presentation orders were used equally often with each exposure duration, and equal numbers of four-, five-, and six-letter words were used with each exposure duration and presentation order combination. Within these constraints, the different conditions were presented in random order within each trial block.

All other aspects of the method were the same as in Experiment 1.

\section{Results and Discussion}

The mean proportions of "longer" responses for words and nonwords are shown at the bottom of Table 1 for both the 33-msec and 67-msec exposure duration conditions. As was the case for the 50-msec exposure duration used in Experiment 1, when the stimulus displays were presented for $67 \mathrm{msec}$, words were judged as longer than nonwords $[f(19)=4.97, p<.001]$. In addition, the magnitude of the familiarity effect was significantly larger for the 67-msec presentation relative to the 50 -msec presentation used in Experiment $1[t(19)=3.22, p<.01]$. However, as suggested by an inspection of Table 1 , when the exposure duration was $33 \mathrm{msec}$, no significant differ- ence was found between the proportion of "longer" responses for words and nonwords $[t(19)<1]$.

Considered together, the results of Experiments 1 and 2 demonstrate that increases in exposure duration simply increase the magnitude of the positive relation between stimulus familiarity and perceived duration. Given these results, it remains unclear as to what constitutes the critical methodological difference distinguishing studies that indicate stimulus familiarity and perceived duration are positively correlated (e.g., Devane, 1974; Warm \& McCray, 1969; Witherspoon \& Allan, 1985) and the studies reported by Avant and his colleagues (Avant \& Lyman, 1975; Avant et al., 1975; Avant \& Thieman, 1985), which demonstrate a negative correlation between stimulus familiarity and perceived duration.

In spite of this inconsistency across studies, the fact that the present studies convincingly demonstrate a positive relation between stimulus familiarity and perceived duration indicates that perceived duration, as measured in the present studies, provides a sensitive indirect measure of stimulus familiarity. More specifically, the present experiments indicate that judgments of perceived duration provide an index of an observer's ability to discriminate between words and nonwords, even when the word/ nonword discrimination is not part of the task instructions. Therefore, by definition, perceived duration provides a sensitive indirect measure of lexical status.

\section{EXPERIMENT 3}

The results of Experiments 1 and 2 indicate that the present methodology can be used to compare the relative sensitivity of direct and indirect measures of lexical status. Given that the indirect duration judgment measure was insensitive to lexical status when the words and nonwords were presented at an exposure duration of $33 \mathrm{msec}$, the present experiment was designed to establish whether a comparable direct measure would show greater sensitivity to the lexical status of stimuli presented at this exposure duration.

\section{Method}

Subjects. Forty undergraduate students at the University of Waterloo participated in the experiment. Each subject participated in two sessions and was paid $\$ 10$ at the completion of the second session. All subjects had normal or corrected-to-normal vision.

Materials and Design. A total of 144 words was selected from the pool of words used in Experiment 1. The selected words had the higher word frequencies, and the minimum word frequency of any word used in this experiment was $\mathbf{8 8}$ occurrences/million. In addition, equal numbers of four-, five-, and six-letter words were selected.

Each subject was tested in two experimental sessions that differed as to the decision required following the presentation of each stimulus sequence. Each session involved 144 trials, and on each trial in each session, one stimulus display contained a randomly selected word and the other display contained a nonword variant of the same word. In one session, the subjects were required to decide which 
stimulus display contained a word (i.e., a direct discrimination of lexical status), and in the other session, the subjects were required to decide which stimulus display was longer in duration. Half of the subjects made "duration" decisions in the first session and "word" decisions in the second session, whereas the order of decisions across sessions was reversed for the remaining subjects.

In each session, the experimental trials were presented in six blocks of 24 trials. The two possible presentation orders for word and nonword displays, as well as the number of four-, five-, and six-letter words used in each condition, were completely counterbalanced within each trial block. Within these constraints, the order of presentation for the different conditions in each trial block was random.

Procedure. All subjects were tested individually in two separate sessions scheduled on 2 consecutive days. Each session lasted approximately 30 to $45 \mathrm{~min}$. Prior to the beginning of each session, the subjects were instructed that they would see two "flashes." In addition, depending on which condition was being tested in the session, the subjects were instructed to decide either "which flash was longer in duration" or "which flash contained a word."

All stimulus displays were presented for $33 \mathrm{msec}$ under presentation conditions that were exactly the same as those used in Experiments 1 and 2.

Immediately after a decision was made on each trial, the subjects were required to rate their confidence in the correctness of the decision. ${ }^{2}$ A confidence scale was displayed on the monitor, and confidence was indicated by moving a cursor along a horizontal line with nine vertical lines placed at equal intervals. The cursor always appeared initially at the center location on the scale, and the subjects moved the cursor either to the left toward a minus sign or to the right toward a plus sign by pressing one of the plates on the right side of the table. Approximately 3,000 msec after the confidence judgment was made, the signal indicating that the computer was ready to display the next trial sequence was presented.

\section{Results and Discussion}

The mean proportion of "longer" responses on the duration judgment task and the mean proportion of "word" responses on the lexical decision task are shown at the top of Table 2. The most important aspect of these data is that the direct lexical decisions were more sensitive than were the indirect duration judgments to the word/nonword distinction. In fact, duration judgments did not discriminate significantly between words and nonwords $[t(39)=1.30, p>.05]$. This null sensitivity for duration judgments is similar to the null effect found in Experiment 2 when the stimuli were presented for $33 \mathrm{msec}$. On the other hand, in contrast to the null sensitivity for duration judgments, accuracy of direct lexical decisions in the present experiment was significantly better than chance $[t(39)=3.23, p<.01]$.

To compare the sensitivity of the two tasks directly, we further analyzed the results using a $2 \times 2$ analysis of variance that evaluated both task (i.e., lexical decision vs. duration judgment) and task order (i.e., lexical decision first vs. duration judgment first). Only the main effect of task was significant $[F(1,38)=5.10, p<.05]$, indicating that performance on the lexical decision task was significantly better than performance on the duration judgment task. These results thus provide strong support for the conclusion that the direct measure of lexical status has greater absolute sensitivity than the comparable indirect duration judgment measure.

\section{EXPERIMENT 4}

The purpose of this experiment was to compare the relative sensitivity of direct and indirect measures using another stimulus dimension. In Experiment 1, the filledduration illusion was successfully replicated in that duration judgments were found to discriminate consistently between filled and blank intervals. In addition, duration judgments were considerably more sensitive to the filled/blank distinction than to the word/nonword distinction. Given this sensitivity of duration judgments to a filled/blank discrimination, it is possible that duration judgments may provide a more sensitive measure of stimulus detection than a comparable direct measure. Thus, the present experiment was designed to compare the relative sensitivity of the indirect duration judgment measure and a comparable direct measure of stimulus detection.

\section{Method}

Subjects. Twenty undergraduate students at the University of Waterloo participated in the experiment and received $\$ 10$ following the completion of two experimental sessions. All subjects had normal or corrected-to-normal vision.

General. This experiment was very similar to Experiment 3. The major difference concerned the decisions that the subjects were required to make in each experimental session. Prior to the beginning of each session, the subjects were told that they would see two "flashes." Depending on the condition being tested in the session, the subjects were instructed either to decide "which flash con-

Table 2

Mean Proportions of Responses in Experiments 3 and 4

\begin{tabular}{ccccc}
\hline Experiment & \multicolumn{1}{c}{ Task } & Response & \multicolumn{2}{c}{ Comparison } \\
\hline 3 & Lexical Decision & "Word" & \multicolumn{2}{c}{ Word vs. Nonword } \\
3 & Duration Judgment & "Longer" & .539 & .461 \\
& & & & .492 \\
4 & Stimulus Detection & "Letters" & \multicolumn{2}{c}{ Word vs. Blank } \\
4 & Duration Judgment & "Longer" & .892 & .108 \\
& & & \multicolumn{2}{c}{ Nonword vs. Blank } \\
4 & Stimulus Detection & "Letters" & .889 & .111 \\
4 & Duration Judgment & "Longer" & .599 & .401 \\
\hline
\end{tabular}


tained a string of letters," or to decide "which flash was longer in duration."

On each of the 144 trials in each experimental session, one display contained a randomly selected word or nonword and the other display consisted of a blank field. Word-blank pairs and nonwordblank pairs occurred equally often. As in Experiment 3, the experimental trials were presented in six blocks of 24 trials, and within each trial block, presentation order and word length were completely counterbalanced.

All other aspects of the method were the same as in Experiment 3.

\section{Results and Discussion}

The mean proportions of "letters" responses on the stimulus detection task and "longer" responses on the duration judgment task are shown in Table 2 . As suggested by an inspection of the table, overall performance, collapsed across the word/nonword dimension, exceeded a chance level on both the detection task $[t(19)=14.01$, $p<.001]$ and the duration task $[t(19)=3.14, p<.01]$. In addition, a $2 \times 2 \times 2$ analysis of variance that evaluated task (i.e., stimulus detection vs. duration judgment), stimulus pairing (i.e., word-blank vs. nonword-blank), and task order (i.e., stimulus detection first vs. duration judgment first) revealed that only the main effect of task was a significant source of variance $[F(1,18)=48.32$, $p<.001]$. Given the greater sensitivity of the detection measure, the present results support the same conclusion as the results of Experiment 3; namely, a direct measure provides a more sensitive index of perception than does a comparable indirect measure based on judgments of perceived temporal duration.

\section{GENERAL DISCUSSION}

The comparisons in Experiments 3 and 4 between the indirect temporal duration judgment task and the direct lexical decision and stimulus detection tasks illustrate the general approach we propose for the study of perception without awareness. According to the logic of this approach, unconscious perception is demonstrated whenever an indirect measure of perception shows greater sensitivity to a stimulus dimension than does a comparable direct measure. Given that the sensitivity of the indirect measure in both studies was less than the sensitivity of the comparable direct measures, the present results are inconclusive with regard to unconscious perception. This is the case since it cannot be determined if performance on the detection and lexical decision tasks was mediated by conscious, unconscious, or both conscious and unconscious processes. In spite of these inconclusive results, the present findings have direct implications for the conclusions reached previously by Avant and Thieman (1985). In addition, the approach illustrated by the present experiments provides a framework for conducting more definitive future studies exploring unconscious processes.

One very straightforward implication of the present results is that they provide absolutely no support for Avant and Thieman's (1985) claim that judgment of apparent visual duration constitutes a sensitive measure of uncon- scious perception. Although Avant and Thieman found that indirect temporal duration judgments were a more sensitive index of perceptual processing than direct forcedchoice recognition judgments, they did not measure these direct and indirect indexes of perception under comparable experimental conditions. In contrast, our findings suggest that when experimental conditions are equated across measures, the sensitivity of direct measures is greater than the sensitivity of comparable indirect measures based on perceived temporal duration. Although this conclusion may be limited to the display and stimulus parameters used in the present studies, the proposed approach provides a method for unambiguously assessing the sensitivity of the temporal duration judgment task relative to the sensitivity of comparable direct measures under a wide variety of experimental conditions.

Another difference between the present findings and the results reported by Avant and Thieman (1985) concerns the direction of the relation between stimulus familiarity and perceived duration. Contrary to the negative relation found by Avant and Thieman, the results of Experiments 1 and 2 indicate a positive correlation between stimulus familiarity and perceived duration. Our results are completely consistent with the results reported by many other investigators (e.g., Devane, 1974; Warm \& McCray, 1969; Witherspoon \& Allan, 1985), whereas Avant and Thieman's results are similar to those reported previously by Avant (e.g., Avant \& Lyman, 1975; Avant et al., 1975). Given that we defined stimulus familiarity (i.e., word vs. nonword) in the same way that Avant and Thieman did and we used a similar comparative time judgment task, this inconsistency in the direction of the observed relation between stimulus familiarity and perceived duration is even more striking. Clearly, additional studies are required to isolate the critical methodological factors responsible for these different patterns of results.

At a more general level, the present experiments illustrate an approach or framework for investigating unconscious processes that has the potential to provide more definitive evidence regarding the validity of unconscious perception. Although the present results, as well as many other empirical findings, are entirely consistent with Eriksen's (1960) earlier conclusion that direct verbal measures are as sensitive indicators of perception as are any indirect measures that have been studied, it is premature to conclude that direct measures are always more sensitive than comparable indirect measures. Before such a conclusion is justified, additional comparisons between a variety of comparable direct and indirect measures are required, and we suggest that the proposed approach provides a conceptual/methodological framework for making such comparisons.

One indication of when indirect indexes may be more sensitive than comparable direct indexes comes from studies of unconscious memory. Although memory is sometimes considered to represent a potential confound in studies of unconscious perception (e.g., Holender, 1986), the boundary between perception and memory is 
necessarily arbitrary, and it is possible to argue that most, if not all, perceptual tasks have a memory component (see Erdelyi, 1986). In fact, if perception and memory are regarded as a continuum rather than a dichotomy, then the proposed approach to the study of unconscious perception is directly applicable to the study of unconscious memory.

One finding indicating that an indirect measure of memory is more sensitive than a comparable direct measure was originally reported by Kunst-Wilson and Zajonc (1980). In this study, Kunst-Wilson and Zajonc presented subjects with 10 irregular octagons, with each octagon being exposed five times for $1 \mathrm{msec}$. Following these initial exposures, the subjects were presented with 10 pairs of octagons, one new and one old, and were required either to indicate which member of each pair had been presented previously (a direct measure of memory) or to choose the octagon in each pair that they liked better (an indirect measure of memory). Performance on the indirect preference measure was $60 \%$ correct, whereas performance on the direct recognition test approximated a chance level of accuracy $(\mathbf{4 8 \%})$. This basic finding has been replicated by several other investigators (e.g., Bonnano \& Stilling, 1986; Seamon, Marsh, \& Brody, 1984). In addition, Mandler, Nakamura, and Van Zandt (1987), using a similar procedure, found that a variety of tasks involving indirect discriminations (i.e., preference, brightness, and darkness) are all more sensitive than a comparable forced-choice recognition task. Taken together, these results constitute strong evidence for unconscious memory, as the consistent finding across studies is that indirect measures of memory are more sensitive than comparable direct measures. Whether similar demonstrations of dissociations between direct and indirect indexes of perception are possible is an open question. However, comparisons of the sensitivity of comparable direct and indirect measures at different delays following stimulus presentation might prove valuable in providing an answer to this question.

Finally, to anticipate a potential misinterpretation of the position developed in this paper, we are not proposing that the only valid or profitable way to investigate perception without awareness is to compare the relative sensitivity of direct and indirect measures. Rather, given the dominance of approaches that basically involve comparing the sensitivity of direct and indirect measures (see Erdelyi, 1986; Holender, 1986), the present paper is an attempt to provide a systematic consideration of the conceptual and methodological issues underlying this type of study. We agree completely with Erdelyi (1986) that methodological rigor cannot replace theoretical development in the study of the unconscious. Indeed, due to the complex nature of this research area, it is essential to state explicitly the methodological implications of theoretical assumptions. In the absence of such clarity, the only option is a futile debate between "believers" and "nonbelievers" that has very little to do with empirical evidence.

\section{REFERENCES}

Allan, L. G. (1979). The perception of time. Perception \& Psychophysics, 26, 340-354.

AvaNT, L. L., \& LMaN, P. J. (1975). Stimulus familiarity modifies perceived duration in prerecognition visual processing. Joumal of Experimental Psychology: Human Perception \& Performance, 1, 205-213.

AVANt, L. L., Lyman, P. J., \& ANTES, J. R. (1975). Effects of stimulus familiarity upon judged visual duration. Perception \& Psychophysics, 17, 253-262.

Avant, L. L., Thieman, A. A. (1985). On visual access to letter case and lexical/semantic information. Memory \& Cognition, 13, 393-404

BALotA, D. A. (1983). Automatic semantic activation and episodic memory. Journal of Verbal Leaming \& Verbal Behavior, 22, 88-104.

Bernstein, I. H., Vyas, A., Bissonnette, V., \& Barclay, P. (1987). Semantic processing: Subliminal perception or context? Bulletin of the Psychonomic Society, 25, 335. (Abstract)

Bonnano, G. A., Stilling, N. A. (1986). Preference, familiarity, and recognition after repeated brief exposures to random geometric shapes. American Journal of Psychology, 99, 403-415.

BOWERS, K. S. (1984). On being unconsciously influenced and informed. In K. S. Bowers \& D. Meichenbaum (Eds.), The unconscious reconsidered (pp. 227-272). New York: Wiley.

Cheesman, J., Merikle, P. M. (1984). Priming with and without awareness. Perceprion \& Psychophysics, 36, 387-395.

Cheesman, J., Merikle, P. M. (1985). Word recognition and consciousness. In D. Besner, T. G. Waller, \& G. E. MacKinnon (Eds.), Reading research: Advances in theory and practice (Vol. 5, pp. 311352). New York: Academic Press.

Cheesman, J., Merikle, P. M. (1986). Distinguishing conscious from unconscious perceptual processes. Canadian Journal of Psychology, 40, 343-367

CraIG, J. C. (1973). A constant error in the perception of brief temporal intervals. Perception \& Psychophysics, 13, 99-104.

DEVANE, J. R. (1974). Word characteristics and judged duration for two response sequences. Perceptual \& Motor Skills, 38, 525-526.

Dixon, N. F. (1971). Subliminal perception: The nature of a controversy. London: McGraw-Hill.

Dixon, N. F. (1981). Preconscious processing. Chichester: Wiley.

DUlANY, D. E., JR., \& ERIKSEN, C. W. (1959). Accuracy of brightness discrimination as measured by concurrent verbal responses and GSRs. Journal of Abnormal \& Social Psychology, 59, 418-423.

DunCAN, J. (1985). Two techniques for investigating perception without awareness. Perception \& Psychophysics, 38, 296-298.

ERdelyi, M. H. (1985). Psychoanalysis: Freud's cognitive psychology. New York: Freeman.

ERDELY1, M. H. (1986). Experimental indeterminacies in the dissociation paradigm. Behavioral and Brain Sciences, 9, 30-31.

ERIKSEN, C. W. (1956). Subception: Fact or artifact? Psychological Review, 63, 71-80.

Eruksen, C. W. (1960). Discrimination and leaming without awareness: A methodological survey and evaluation. Psychological Review, 67, 279-300.

Fowler, C. A. (1986). An operational definition of conscious awareness must be responsible to subjective experience. Behavioral \& Brain Sciences, 9, 33-35.

Fowler, C. A., Wolford, G., Slade, R., \& Tassinary, L. (1981). Lexical access with and without awareness. Journal of Experimental Psychology: General, 110, 341-362.

Goldfarb, J. L., Goldstone, S. (1963). Time judgement: A comparison of filled and unfilled durations. Perceptual \& Motor Skills, 16, 376 .

GreenwaLd, A. G. , LIU, T. J. (1985). Limited unconscious processing of meaning. Bulletin of the Psychonomic Society, 23, 292. (Abstract)

HeNLEY, S. H. A. (1984). Unconscious perception re-revisited: A comment on Merikle's (1982) paper. Bulletin of the Psychonomic Society, 22, 121-124. 
Holender, D. (1986). Semantic activation without conscious identification in dichotic listening, parafoveal vision, and visual masking: A survey and appraisal. Behavioral \& Brain Sciences, 9, 1-23.

HUMPHREYS, G. W. (1981). Direct vs. indirect tests of the information available from masked displays: What visual masking does and does not prevent. British Journal of Psychology, 72, 323-330.

KuCERA, H., FranCIS, W. N. (1967). Computational analysis of present-day American English. Providence, RI: Brown University Press.

Kunst-Wilson, W. R., \& Zajonc, R. B. (1980). Affective discrimination of stimuli that cannot be recognized. Science, 207, 557-558.

LazArus, R. S., MCCleary, R. A. (1951). Autonomic discrimination without awareness: A study of subception. Psychological Review, 58, 113-127.

Macmillan, N. A. (1986). The psychophysics of subliminal perception. Behavioral \& Brain Sciences, 9, 38-39.

Mandler, G., Nakamura, Y., \& Van Zandt, B. J. S. (1987). Nonspecific effects of exposure on stimuli that cannot be recognized. Journal of Experimental Psychology: Learning, Memory, \& Cognition, 13, 646-648.

MARCEL, A. J. (1983). Conscious and unconscious perception: Experiments on visual masking and word recognition. Cognitive Psychology, 15, 197-237.

McCauley, C., Parmelee, C. M., Sperber, C. D., \& Carr, T. H. (1980). Early extraction of meaning from pictures and its relation to conscious identification. Joumal of Experimental Psychology: Human Perception \& Performance, 6, 265-276.

MerIKLE, P. M. (1982). Unconscious perception revisited. Perception \& Psychophysics, 31, 298-301.

Merikle, P. M., Cheesman, J. (1986). Consciousness is a "subjective" state. Behavioral \& Brain Sciences, 9, 42-43.

NAvON, D. (1986). On determining what is unconscious and what is perception. Behavioral \& Brain Sciences, 9, 44-45.
PAAP, K. R. (1986). The pilfering of awareness and guilt by association. Behavioral \& Brain Sciences, 9, 45-46.

Purcell, D. G., Stewart, A. L., \& Stanovich, K. E. (1983). Another look at semantic priming without awareness. Perception \& Psychophysics, 34, 65-71.

Seamon, J. G., Marsh, R. L., \& Brody, N. (1984). Critical importance of exposure duration for affective discrimination of stimuli that are not recognized. Journal of Experimental Psychology: Learning, Memory, \& Cognition, 10, 465-469.

STROOP, J. R. (1935). Studies of interference in serial verbal reactions. Journal of Experimental Psychology, 18, 643-661.

Thomas, E. A. C., Weaver, W. B. (1975). Cognitive processing and time perception. Perception \& Psychophysics, 17, 363-367.

Warm, J. S., Greenberg, L. F., \& Dube, C. S., I. (1964). Stimulus and motivational determinants in temporal perception. Journal of Psychology, 58, 243-248.

WARM, J. S., \& MCCRAY, R. E. (1969). Influence of word frequency and length on the apparent duration of tachistoscopic presentations. Journal of Experimental Psychology, 79, 56-58.

WITHERSPOON, D., ALLAN, L. G. (1985). The effect of a prior presentation on temporal judgments in a perceptual identification task. Memory \& Cognition, 13, 101-111.

WolFORD, G. (1986). A review of the literature with and without awareness. Behavioral \& Brain Sciences, 9, 49-50.

\section{NOTES}

1. The visual angle subtended by each stimulus dimension is provided within parentheses following each linear measurement.

2. The confidence ratings did not provide any data relevant to an assessment of the relative sensitivity of the two measures. Thus, these data are not presented or discussed in this paper.

\section{APPENDIX}

a) Given that direct and indirect indices may reflect conscious, unconscious, or both conscious and unconscious processes, the sensitivity of these indices to conscious and unconscious information can be expressed by the following equations:

$\begin{array}{ll}\text { sensitivity of } & \begin{array}{l}\text { sensitivity of direct } \\ \text { direct index }\end{array} \\ \text { index to conscious } \\ \text { information }\end{array}$

$$
(\geq 0)
$$

sensitivity of $\quad$ sensitivity of indirect indirect index $=$ index to conscious information

$$
(\geq 0)
$$

sensitivity of direct

+ index to unconscious information

$$
(\geq 0)
$$

sensitivity of indirect

$+\quad$ index to unconscious information

\section{b) If empirically we find:}

$$
\begin{aligned}
& \text { sensitivity of } \\
& \text { indirect index }
\end{aligned}>\begin{aligned}
& \text { sensitivity of } \\
& \text { direct index }
\end{aligned}
$$

then using the equations in (a):

sensitivity of indirect index to conscious information sensitivity of indirect

+ index to unconscious information sensitivity of direct index to conscious information sensitivity of direct

+ index to unconscious information 
APPENDIX (continued)

c) Subtract from both sides :

sensitivity of indirect index to unconscious information sensitivity of indirect

index to conscious

information

$$
>(
$$
sensitivity of direct index to conscious information

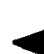

$\left.\begin{array}{l}\text { sensitivity of indirect } \\ \text { index to conscious } \\ \text { information }\end{array}\right)+$
information

sensitivity of direct index to unconscious information

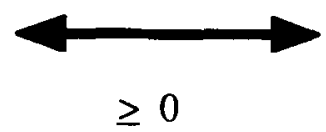

d) Thus, given (a), (b) and (c), we can conclude:

sensitivity of indirect index to unconscious

$>0$

information

(Manuscript received April 11, 1988;

revision accepted for publication July 1, 1988.) 